\title{
Not-so-Minor Injuries: Delayed Diagnosis of a Large Splinter
}

\author{
Lampros Liasis, ${ }^{1,}{ }^{*}$ Lara Howells, ${ }^{1}$ and Harry T. Papaconstantinou ${ }^{2}$ \\ ${ }^{1}$ General Surgery Department, Northwick Park Hospital, London, UK \\ ${ }^{2}$ Baylor Scott and White Memorial Hospital, Temple, Texas \\ "Corresponding author: Lampros Liasis, General Surgery Department, Northwick Park Hospital, Consultant Laparoscopic General Surgeon, Hon Senior Clinical Lecturer, \\ Imperial College School of Medicine, Watford Rd, Harrow, Middlesex HA1 3UJ, London, UK. Tel: +44-7580834455, E-mail: lampros.liasis@nhs.net
}

Received 2015 September 23; Revised 2015 December 28; Accepted 2016 January 25.

\begin{abstract}
Introduction: In contrast with victims of major trauma, patients who suffer minor injuries receive little specialist input. In most cases, this causes no difficulty, but there are situations where minor trauma results in persistent disability affecting the quality of life.

Case Presentation: A young man sustained a perineal puncture wound resulting from a fall onto a bush. Following an initial delay, he sought medical advice for a continual pain in his right leg, and a discharging perineal wound. A computed tomography (CT) scan and flexible sigmoidoscopy failed to identify the cause, and he was subsequently discharged from hospital. One year after his initial presentation, a magnetic resonance imaging (MRI) scan identified a retained foreign body consistent with a fragment of wood.

Conclusions: Penetrating trauma from wooden fragments provides a diagnostic challenge. A stubborn discharge from a wound must always raise the suspicion of retained fragment. Early and appropriate surgical exploration is imperative.
\end{abstract}

Keywords: Minor, Injury, Trauma, Delay, Wood

\section{Introduction}

In the USA, major trauma - most often due to road accidents - is the leading cause of death and disability amongst people under age forty. Its victims normally receive high quality care in designated specialist trauma centres staffed by experienced teams. Patients suffering from minor injuries, by contrast, receive little specialist input. In most cases, this causes no difficulty, and the Major Trauma Call Activation Guidelines are in place to identify the full range of activation criteria. There are, nonetheless, situations where minor trauma causes continuing disability affecting quality of life. We describe such a case and its diagnostic challenges.

\section{Case Presentation}

Our patient, a 21 years old man, sustained a perineal puncture wound resulting from a fall of eight feet onto a bush whilst attempting to access his property through a window. Initially, he experienced only mild pain in his right leg, and did not seek medical advice. The pain in his leg, although not severe, persisted and a week later he consulted his primary care physician. The physical findings then were a few scratches on his right buttock and the adjacent perineal region. In addition, however, he had what appeared to be a healing perineal puncture wound measuring $0.5 \mathrm{~cm}$. He was provided with some dressings, analgesic tablets, and advice to return should the pain fail to resolve in a week or so. Two weeks later, he again consulted his GP because of a discharge from the perineal puncture wound. Pain was not a feature. Re-examination revealed a small sinus at the site of the wound with a serous discharge. A swab was taken for microbiology, culture and sensitivity, and the patient was started on an oral antibiotic. As the discharge continued, despite a several weeks course of the antibiotic, the patient was referred for a surgical opinion. At the surgical clinic, the presences of a foreign body, or a fistula with the ano-rectal canal were viewed as possibilities. A pelvic CT scan demonstrated a sinus tract extending from the anus to the right obturator internus muscle, but there was no evidence of a retained foreign body. A flexible sigmoidoscopy and wound exploration were performed under general anaesthesia, but revealed no significant findings. The wound was debrided, thoroughly washed out, and the patient discharged from hospital the following day. Despite adequate wound care, and continuous treatment with antibiotics, the discharge continued. Six months later, the patient was referred to the colorectal surgery department for further investigation. At that point, examination showed a chronic perianal wound to the right of the anus with a blood-stained discharge. A repeat CT scan (Figure $1 \mathrm{~A}$ and $\mathrm{B}$ ), and a MRI (Figure $1 \mathrm{C}$ and D) of the pelvis showed a foreign body with an associated abscess and inflammatory fat located within the right hemipelvis. When an elective preperitoneal pelvic exploration was carried out, we found a chronically inflamed, pus-filled cavity in the right hemipelvis, which was push- 
ing and displacing the bladder anteriorly. A foreign body was identified and removed (Figure 2). The pathology report indicated that the specimen was consistent with a fragment of wood. The patient was discharged two days later and made a complete recovery.

\section{Discussion}

Our case illustrates how, what at first presents as minor trauma, can lead to serious complications affecting the quality of life. Reflecting on the circumstances, we tried to fathom why the diagnosis had been delayed for more than a year. Was it, for example, a fault in a system where the management of minor trauma is in the hands non-specialists? Was it simply a misdiagnosis, or was there something unique in the case that could provide a lesson?

Serious penetrating trauma for example by knife or gunshot, is often attended by drama and media coverage, and ranks as a top priority for the trauma surgeon. The victims of such injuries usually receive high quality care in large Level I Trauma Centres, where specialized teams, supported by state-of-the art equipment, can offer the best possible outcomes.

By contrast, minor penetrating trauma by a wood fragment usually occurs in less colourful circumstances, and patients more likely to seek medical help in smaller, local, health facilities managed by non-specialists. Our patient delayed seeking medical advice until a week after his fall, and even then, his main concern was pain in his right leg, which conceivably diverted attention from the more significant perineal lesion. Moreover, a review of the medical record of the initial consultation showed no hint of the potential problems. His subsequent discharge from the primary care situation, and the eventual referral to the colorectal surgeon, were appropriate. The initial CT scan had failed to demonstrate a foreign body. Even a careful review of this scan failed to show any suspicion of a foreign body.

Penetration by a wooden object carries the risk of leaving residual fragments in the body that can be overlooked at routine physical examination and imaging practice (1, 2 ). As the densities of fragments of wood approximate to those of serous fluid and normal soft-tissue, they can be difficult to identify (3). The initial CT scan in our case failed to do so and the repeat CT scan able to do so only because the fragment had become calcified.

We reviewed the literature to find out whether a method exists that would have facilitated earlier diagnosis in our case. A high level of clinical suspicion is the first priority. Persistent sinus tract drainage is an indicator of the presence of a foreign body, and demands early exploration if serious sequelae are to be avoided $(1,4)$. In our case, despite exploration of the wound, we were unable to identify the foreign body intraoperatively. Additional diagnostic tests can provide valuable information. Ultrasonography is an option, as wooden foreign bodies generate a detectable echogenic source of acoustic shadowing $(3,5)$. Its role, however, is relatively limited. It is skilled operatordependent, and cannot be used everywhere in body. MRI is the only available imaging technology that can help with the diagnosis from the outset, and remains the technique of choice in situations where there is high suspicion of retained foreign body (1).

Patients with minor traumatic lesions are often subject to misdiagnosis. When seeking medical help, they do not meet the activation criteria for trauma call, and are often treated by non-specialists. Moreover, even after careful physical examination and highhtened clinical suspicion, it is easy to overlook complications related to minor, injuries. Is it possible to prevent these undesirable sequelae, or should we concede that the problem offers no practicable solution?

The answer to these questions may rest among the primary principles of trauma care. Trauma patients experience a sudden attack on their health, and will subsequently undergo a natural healing process. Those with minor injuries usually take a short time to return to normality. Any delay in the healing process should raise the suspicion of some overlooked factor. Penetrating trauma with wooden or other material can remain unrecognised by routine imaging. What is important is that, if a minor injury fails to heal as expected, we should refer the patient to a specialist. Advanced diagnostic techniques - such as MRI - may be the only way to establish a diagnosis. Accurate evaluation of minor injuries, while lacking the urgency of major trauma, can be crucial to the quality of life of the injured patient.

\subsection{Conclusion}

Penetrating trauma from wooden fragments provides a diagnostic challenge for the non-trauma physician. A persistent discharge from a wound must always raise a suspicion of retained fragment. MRI is the imaging study of choice. Early and appropriate surgical exploration is crucial, as late sequelae can be calamitous.

\section{References}

1. Gul S, Dusak A, Songur M, Kalayci M, Acikgoz B. Penetrating spinal injury with a wooden fragment: a case report and review of the literature. Spine (Phila Pa 1976). 2010;35(25):E1534-6. doi: 10.1097/BRS.0b013e3181d9b7f8. [PubMed: 21102287].

2. Paul AM, Grundmann T. [Intraorbital wooden foreign body undetected on CT]. HNO. 2010;58(12):1237-40. doi:10.1007/s00106-010-2192-9. [PubMed: 21085921]. 

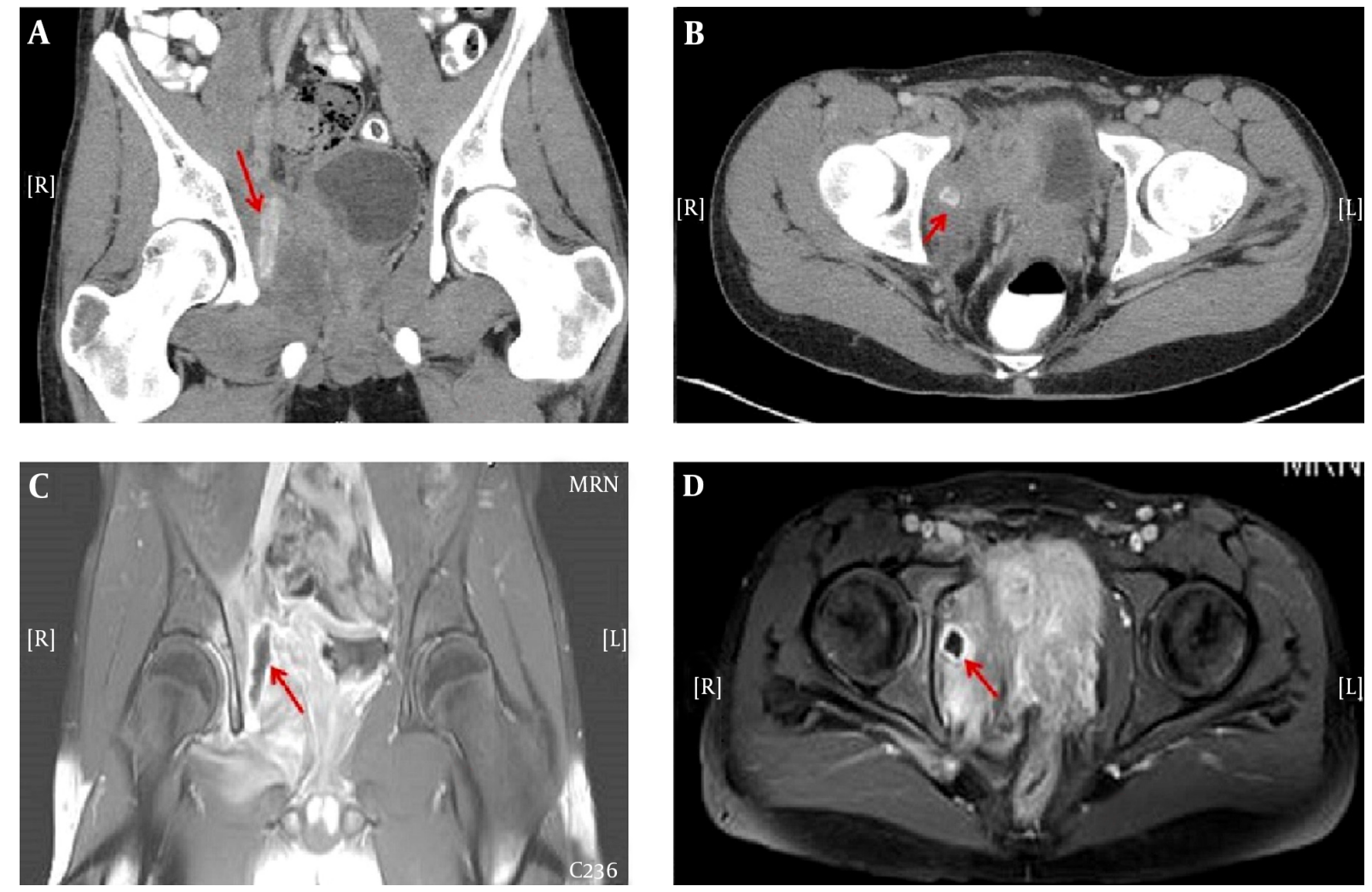

Figure 1. A repeat A and B, CT scan; and C and D, MRI of the pelvis.

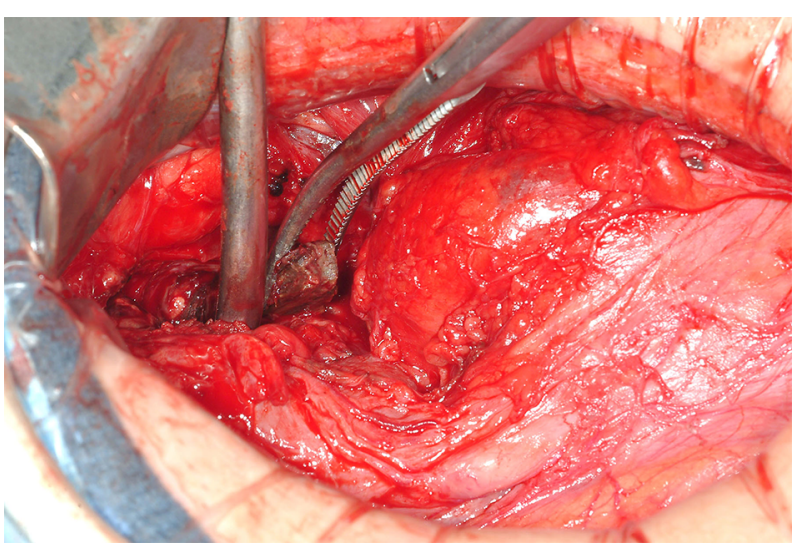

3. Ginsburg MJ, Ellis GL, Flom LL. Detection of soft-tissue foreign bodies by plain radiography, xerography, computed tomography, and ultrasonography. Ann Emerg Med. 1990;19(6):701-3. [PubMed: 2188542].

4. Rao J, Messahel A, Grimes K, Sanders K. Elusive penetrating foreign body to the neck causing partial epiglottic airway obstruction. $J$ Craniomaxillofac Surg. 2011;39(1):37-9. doi: 10.1016/j.jcms.2010.03.004. [PubMed: 20456968].

5. Rockett MS, Gentile SC, Gudas CJ, Brage ME, Zygmunt KH. The use of ultrasonography for the detection of retained wooden foreign bodies in the foot. J Foot Ankle Surg. 1995;34(5):478-84. doi: 10.1016/S10672516(09)80024-0. [PubMed: 8590883] discussion 510-1.

Figure 2. The Pathology Report 